\title{
UN BON MILLÉSIME: mariage de la photonique et du vin
}

\author{
Patrick CHABASSIER ${ }^{1}$ \\ Gilles BRIANCEAU \\ Pôle de compétitivité \\ Route des Lasers \\ p.chabassier@routedeslasers.com \\ Bordeaux Aquitaine INNO'VIN \\ gilles.brianceau@innovin.fr
}

À Bordeaux, le vin est un sujet d'importance. Et chaque année se déroule à son rythme. Le pôle Route des Lasers a su rapidement prendre en compte cette composante régionale et l'intégrer avec modération dans ses principes de fonctionnement... Mais c'est sans modération que le cluster Inno'vin et Route des Lasers ont établi depuis près de deux ans une collaboration régulière visant à mettre en relation des experts des deux domaines. Un accord a été signé en 2014 pour concrétiser ce partenariat.

qui fédère au sein de l'université de Bordeaux l'ensemble des forces de recherche et de formation sur la vigne et le vin.

C'est dans le but de faire émerger de nouveaux projets et surtout de donner à nos membres respectifs l'occasion de prendre pied sur un nouveau secteur d'application que nous avons donc organisé des évènements de rencontre VVOP (Vigne, Vin, Optique et Photonique) entre les professionnels de la vigne et du vin et des professionnels et experts de la filière photonique du pôle Route des Lasers. Ces rencontres sont très productives, se déroulent sur une demi-journée et sont centrées sur une ou deux thématiques qui permettent de mobiliser les bons experts des deux domaines et de les faire réfléchir ensemble. Comme souvent à Bordeaux, un moment de convivialité permet de favoriser des rencontres plus informelles...

Des groupes de travail naissent de ces rencontres et ont pour objectif de détecter l'émergence de besoins, de technologies disponibles et de projets collaboratifs permettant de les associer. Ces projets sont ensuite suivis et soutenus par les animateurs du pôle et du cluster afin de trouver les partenaires et le financement les mieux adaptés. Plusieurs axes de travail sont d'ores et déjà explorés.

\section{La lutte contire les maladies de la vigine}

Lorsque le printemps se réveille dans le domaine viticole, la nature reprend vie. Elles ont pour nom Mildiou, Odium, Botrytis, Black Rot, Flavescence dorée etc. et s'attaquent au feuillage ou aux grappes en pleine croissance ou à maturité... Les maladies sont les ennemies du vignoble et peuvent aller jusqu'à la destruction de parcelles entières de ceps productifs ou la détérioration de la qualité des raisins. C'est le sujet de préoccupation majeur des viticulteurs qui ont déjà à leur disposition des systèmes d'alertes géographiques basés sur des mesures météo et des statistiques locales. L'épandage de produits de traitement phytosanitaires sur la totalité des parcelles reste la solution usuelle pour contrer la survenue des maladies. Ce n'est pas la solution d'avenir.

On peut se référer au site de l'Institut Français de la Vigne et du Vin 
(IFV, centre de recherche et expérimentation national) pour comprendre le principal sujet: «Les conclusions du Grenelle de l'environnement ont fait émerger un consensus sur la nécessité d'une politique ambitieuse de réduction de l'usage des produits phytosanitaires. Les plans Ecophyto qui en résultent visent ainsi à diminuer l'usage des pesticides et à supprimer progressivement l'utilisation des molécules présentant les risques les plus forts. Dans ce contexte, l'objectif est d'accompagner la prise de risque liée aux changements de pratiques et de systèmes culturanx, pour une viticulture économiquement viable, qualitative et plus respectueuse de l'environnement et moins dépendante des produits phytosanitaires.»

De nombreuses recherches actuelles sont donc centrées sur l'anticipation de la détection des maladies afin de traiter à faibles doses des secteurs bien déterminés sur lesquels on a eu la possibilité de détecter un précurseur de la maladie. Dans ce domaine, l'optique photonique peut intervenir de manière efficace.

Par la vision qui permet de travailler dans une large gamme spectrale et de détecter les symptômes les plus précoces de l'apparition des maladies sur le feuillage des vignes.
L'IMS de Bordeaux travaille sur ces sujets depuis de nombreuses années et s'attache à classifier les différentes maladies identifiables par une signature personnalisée. Les travaux se font en relation avec les équipes de l'IFV et de l'INRA pour rendre ces technologies applicables sur le terrain. On imagine des solutions de capteurs embarqués sur le tracteur (enjambeur) capables de réaliser cette détection lors des épandages et de déclencher la juste dose de produit de manière locale.

Par la fluorescence active ou la spectroscopie qui vont s'intéresser aux précurseurs biologiques, physiques ou chimiques des maladies. Des sociétés comme Force-A sont à la pointe de ce progrès avec des appareils à main utilisables par les viticulteurs sur le terrain pour la détection du Mildiou.

\section{La culture de la vigne (croissance, maturité, vigueuric)}

Le métier de viticulteur nécessite beaucoup de vigilance. Tout au long de l'année, celui-ci observe et intervient dans le vignoble. En premier, il y a le sol: Médoc, Graves, Sauternais,

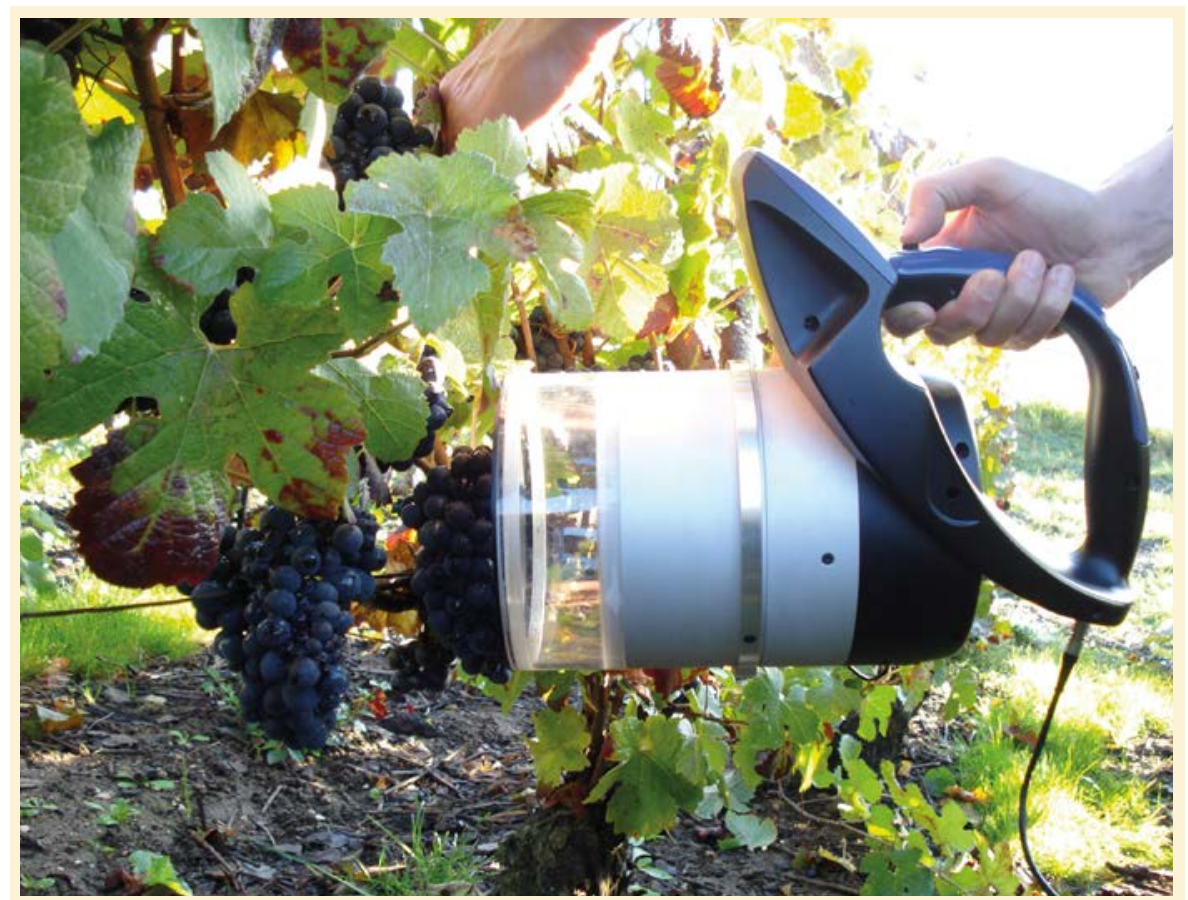

Force-A propose une gamme de capteurs optiques utilisables sur le terrain.

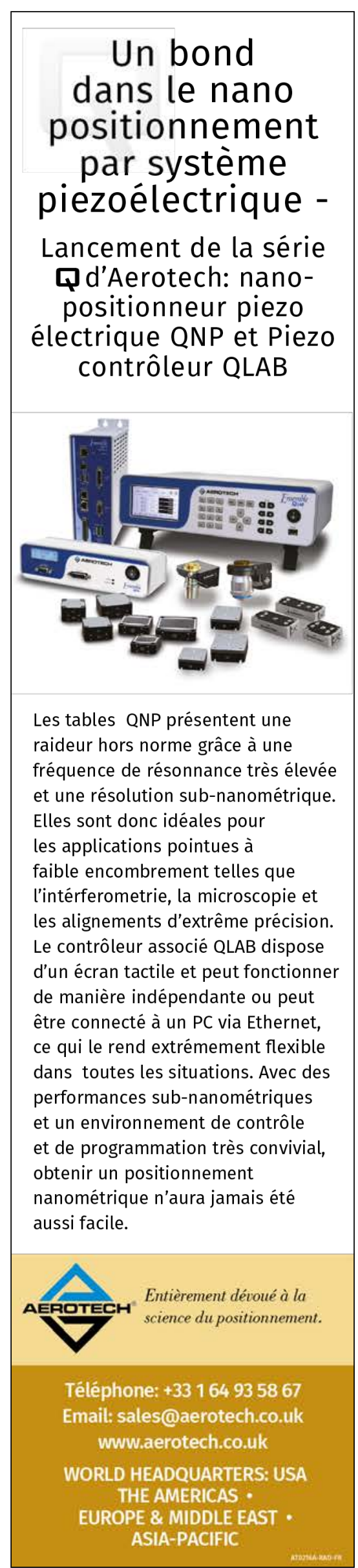


Entre-deux-Mers, Libournais, Blayais et Bourgeais... Partie essentielle du terroir, c'est un héritage de tant de siècles et de sueur que la main de l'homme a su dompter pour produire le meilleur. Cette terre est vivante et il faut la connaître pour la respecter et lui donner les soins nécessaires. Ici, le laser intervient pour effectuer des analyses spectroscopiques par méthode LIBS qui nous renseignent sur la composition physico-chimique de la parcelle et la cartographie de manière très fine. Ainsi, on saura planter, surveiller la croissance et apporter les soins en fonction des caractéristiques du sol.

Sur cette terre pousse la vigne. Leurs cépages fondent la richesse de nos vins qui s'assemblent à la baguette de notre culture. On les nomme Merlot, Cabernet, Sauvignon, Petit verdot, Sémillon, Muscadelle... et leurs qualités se combinent pour tendre à l'harmonie. C'est ici le domaine de la vision. Avec l'émergence de nouveaux capteurs et de traitement d'images adaptés, on peut tirer bénéfice d'un large domaine spectral: visible, proche infrarouge, infrarouge et même UV et RX... À partir du greffon jusqu'à la fin de vie du cep, l'observation de cette matière vivante permet d'obtenir des informations cruciales sur sa capacité à produire le nectar final.

Les capteurs embarqués sur des véhicules, des drones, des petits avions ou même des satellites donnent une vision complète des parcelles et l'analyse experte de ces images permet de déterminer de nombreux facteurs biophysiques des plants. La société Telespazio est spécialisée dans l'interprétation des images à destination de ses clients. Elle a créé à Bordeaux un «EarthLab » et fournit des données personnalisées sur des parcelles concernant la croissance, la vigueur des plantations et la maturité du raisin. En particulier, de nombreuses solutions basées sur l'analyse de la chlorophylle (capteurs proche infrarouge et visible) existent pour une bonne appréhension de la vigueur d'un vignoble.

L'été se déroule sous surveillance. Le soleil produit le miracle. Les soins donnés à la vigne dépendent de la finesse et de la pertinence des informations disponibles. Les stratégies de conduite des tailles d'éclaircissage peuvent se gérer au pied de vigne près. Les innovations de ce secteur vont venir très rapidement du développement des capteurs hyperspectraux de très haute résolution dans le domaine visible et infrarouge.

\section{EARTHLAB

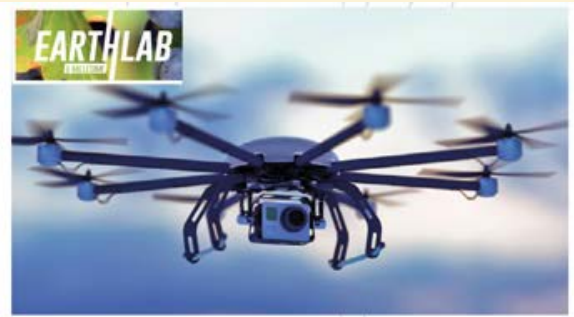

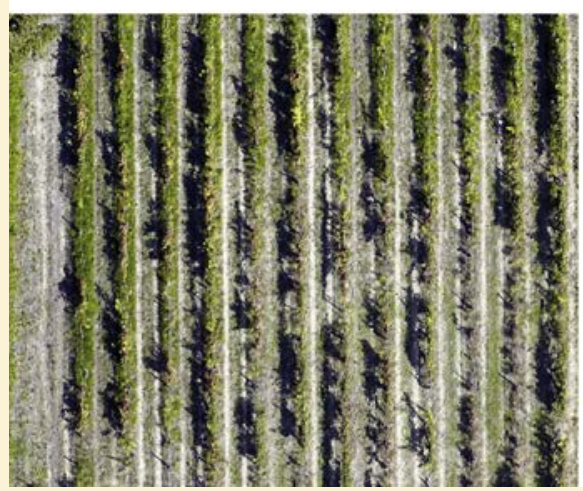

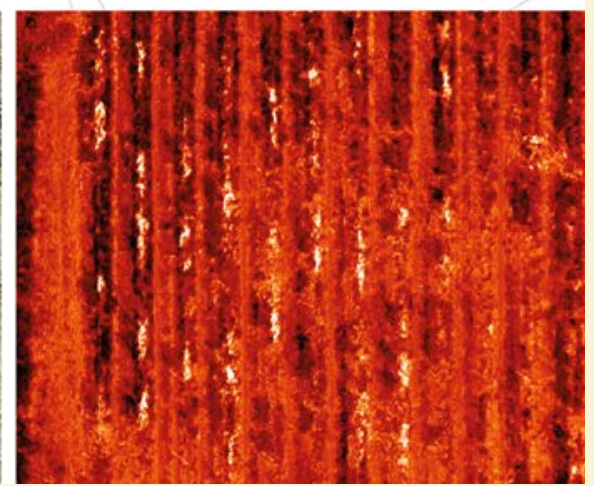

EarthLab offre un niveau de détail extrêmement précis permettant de détecter les variabilités intra-parcellaires.

\section{La vendange}

La fin de l'été est une période très active de la vie de la propriété viticole. Il faut aller très vite car les conditions météorologiques sont souvent le facteur bloquant et critique pour la réussite de l'année. Les machines à vendanger embarquent des systèmes de vision ou d'autres capteurs optiques capables de déterminer la maturité des grappes. On parle alors de récolte sélective.

Jusque-là réservé aux chais, le système perfectionné de tri optique des baies s'invite aujourd'bui à bord de la machine à vendanger, permettant ainsi d'obtenir une précision de tri inégalé, et ce dès la vendange. Le système Opti Grape développé par la société New Holland a reçu à ce titre une médaille d'or au palmarès de l'innovation $d u$ SITEVI 2013.

L'étape suivante sera de trier non seulement les baies sur des critères physiques (baies saines ou pas, corps étrangers...) mais aussi sur des critères de maturité du raisin: dans ce domaine, il est d'ores et déjà possible d'analyser en temps réel sur la machine à vendanger la teneur en anthocyanes des raisins, facteur clé d'évaluation de la maturité. Ce dispositif, mis au point par la société Force-A, met en ceuvre un capteur optique Multiplex et permet d'avoir des informations cruciales pour la suite $d u$ process de vinification. Ce système a lui aussi été récompensé par une citation au palmarès de l'innovation $d u$ SITEVI2013. (Article de Winemak-in, www.winemak-in.com)

Les machines de tri des baies permettent de rendre automatiques des opérations jusque-là manuelles. Il s'agit d'éliminer les intrus ou de sélectionner les grappes les plus mûres ou celles qui n'ont pas de défaut ou de maladie. Ces machines reposent sur des systèmes de vision à très haute cadence, avec des astuces de choix d'éclairage et de bande spectrale qui autorisent des reconnaissances 
avec un faible taux d'erreur. La société Bucher Vaslin (leader mondial des systèmes de tri en viticulture) et le centre technologique bordelais Alphanov ont travaillé ensemble sur le test de différentes techniques de tri optique de baies de raisin à fort débit.

\section{La vinification et L'élevage du vion}

Il se passe aussi beaucoup de choses dans le chai, lorsque le raisin est rentré et que l'automne avance. C'est dans les cuves que le moût se transforme en vin. Les étapes sont complexes et les facteurs environnementaux prennent une grande importance pour l'obtention d'une qualité finale maîtrisée. Le mélange est très inhomogène, sensible à la température et la surveillance des concentrations de certains éléments chimiques, des tanins etc. est nécessaire pour que la vinification se fasse dans de bonnes conditions (ex: thermorégulation de la cuve).

C'est un domaine où les capteurs optiques deviennent d'une grande utilité. On voit apparaitre des capteurs présents dans le laboratoire du chai qui mesurent des taux de gaz dissous, de sucre, des taux d'alcool, de la teinte, de la turbidité... Il y a ici beaucoup à faire pour que la maîtrise de la vinification soit automatisée, l'œil, l'odorat et le palais du maître de chais sont encore garants de la qualité finale, mais l'aide à la décision passe par le développement de nouveaux moyens autour de la cuve. Les derniers progrès permettent d'équiper les cuves et fournissent ainsi un moyen d'enregistrement régulier des paramètres essentiels. La conduite de la cuvaison lors du remontage s'en trouve alors largement facilitée.

L'élevage vient plus tard, comme une période calme qui démarre avec l'hiver. Le vin se repose en fûts pour atteindre doucement la qualité souhaitée. On entre dans le domaine de l'œnologie. De nouveaux instruments fournissent l'analyse du vin basée sur la spectroscopie infrarouge par transformée de Fourier (IRTF). Les nouveaux modèles sont composés d'une cuvette de gaz dans laquelle le $\mathrm{SO}_{2}$ évaporé est mesuré suivant la technologie IRTF.

Les capteurs à fibres optiques sont également de bons candidats pour équiper des cuves de vinification ou des fûts d'élevage du vin sans apporter de risque de contamination, ni de dégradation. Il faut savoir les rendre abordables en prix pour que l'équipement des cuves puisse bénéficier de l'étendue de leurs performances. À terme, nous pourrons voir vieillir le vin au travers de courbes qui se dessinent sur des écrans de contrôle... Cela n'empêchera personne de venir dans la cave pour le déguster en refaisant le monde avec le viticulteur. Les portes de leurs chais sont toujours ouvertes aux amateurs.

\section{In vino veritas}

De nombreuses étapes de la production du vin n'ont pas été évoquées dans cet article. Elles sont toutes très importantes pour que la bouteille que l'on partage entre amis reste un moment d'exception. Une nouvelle génération de capteurs est en marche. On sait qu'elle résultera de la rencontre de l'innovation avec les besoins et progrès souhaités par les exploitants. Il reste des champs libres ouverts à de nouvelles investigations que notre pôle Route des Lasers et le cluster Inno'vin sont prêts à accompagner. Il est certain que l'optique photonique peut y trouver sa place. Cependant, l'aspect économique est une contrainte majeure qu'il faut prendre en compte systématiquement dans ce milieu de grande production, avec des moyens faibles en investissement.

Par l'innovation, nous espérons pouvoir contribuer à améliorer les procédés de production en réduisant leur impact environnemental et sanitaire. Nous espérons donner aux producteurs le moyen de conserver leur fierté de pouvoir produire des vins de qualité sur un terroir exceptionnel. Mais nous le savons, par raison, ou par expérience, il faut consommer avec modération.

\section{COMPONENTS}

small components MASSIVE IMPACT
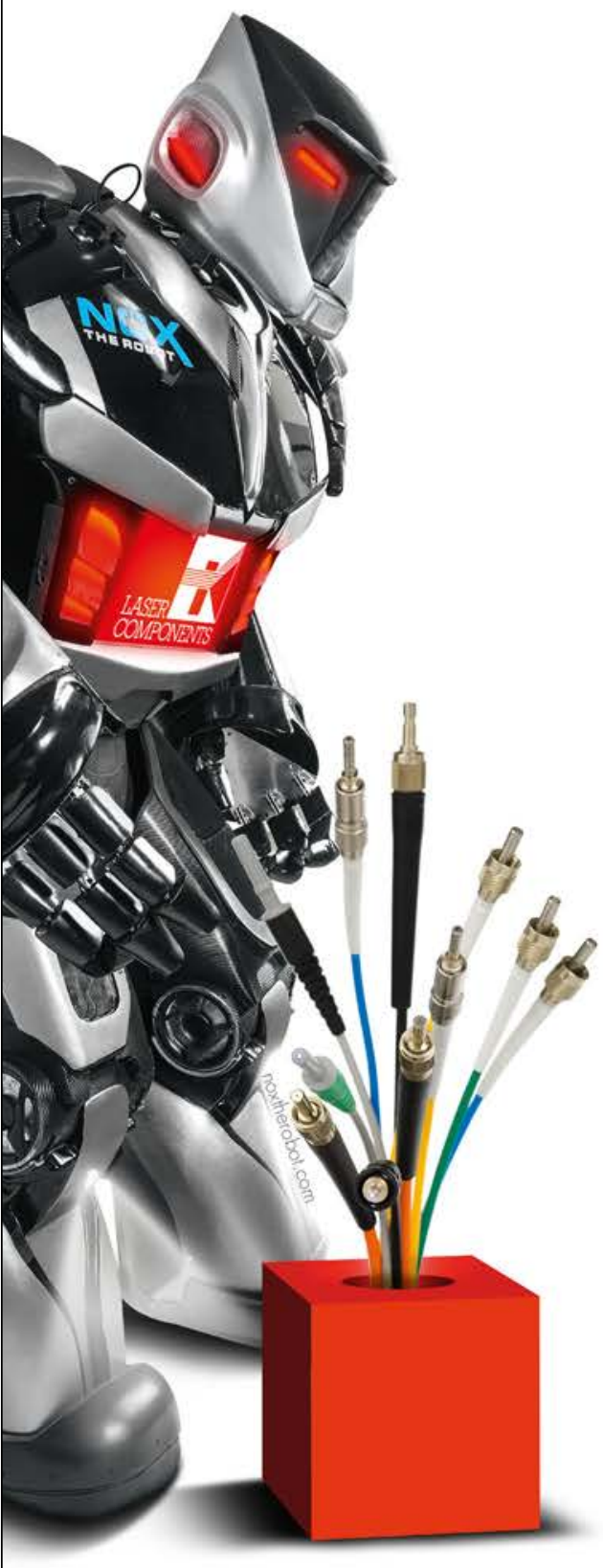

Fiber Optics

- Fiber Optical Couplers

- Collimators and Connectors

- Assembled Fibers 